Internal Biology Review. Volume 1, Issue 1, May 2017

\title{
Diverse insulin-like peptides in Caenorhabditis elegans
}

Authors

Yohei Matsunaga ${ }^{1,2}$

Takashi Iwasaki $^{1}$

Tsuyoshi Kawano ${ }^{1, *}$

Affiliation

${ }^{1}$ Department of Bioresources

Science, The United Graduate

School of Agricultural Sciences,

Tottori University, Tottori

680-8553, Japan.

${ }^{2}$ Present address: Department

of Pathology, Emory

University School of Medicine, GA 1330322, USA.

Email addresses:

YM

yohei.matsunaga@emory.edu

TI

itaka@muses.tottori-u.ac.jp

TK

kawano@muses.tottori-u.ac.jp

Correspondence

Tsuyoshi Kawano (TK)

kawano@muses.tottori-u.ac.jp

\section{Abstract}

Peptide hormones are conserved in living organisms to modulate homeostasis. To elucidate molecular mechanisms in the synthesis, secretion, and functions of peptide hormones, model organisms have been used. Caenorhabditis elegans, one of model organisms, is a good tool since: 1) genome size of the worm is small with over $40 \%$ homology to human genome, 2) numerous genetics methods are available, and 3) the worms are transparent throughout the life cycle, so that the secretion of peptide hormones can be followed at cellular level in living preparations by Green Fluorescent Protein tagged peptides. This review reports the structures, physiological functions, and secretion of insulin-like peptides, one family of peptide hormones, with our latest findings in the model organism, Caenorhabditis elegans. 
Internal Biology Review. Volume 1, Issue 1, May 2017

\section{Diverse insulin-like peptides in Caenorhabditis elegans}

\section{Introduction:}

Most of living organisms can sense change in their environments. To adapt these changes, they change behavior and metabolism to maintain their organismal homeostasis. Hormones are key factors to regulate the homeostasis, and have diverse chemical structures, such as eicosanoids; derivatives of fatty acids like arachidonic acid, steroids; organic compounds with four rings arranged in a specific configuration, and amino acid/protein derivatives. For example, insulin is one of amino acid/protein derivatives, and produced by $\beta$ cells of the pancreatic islets to regulate the metabolism of carbohydrates by promoting the absorption of glucose from the blood into fat, liver, and skeletal muscle cells. Defect of insulin productions results in high blood sugar levels, causing type-1 diabetes. Thus, elucidation of molecular mechanisms of the synthesis and secretion, and functions of hormones, including how to regulate signal transduction is an important subject for further understanding of the metabolism, homeostasis, and diseases.

Researches using one of model organisms, Caenorhabditis elegans, have provided some important information of underlying mechanisms of biological processes by means of genetics. As for study on aging, age-l was first identified by a genetic screen for long-lived animals, ${ }^{1}$ and subsequent analysis suggested that the longevity phenotype in this mutant strain was due to a recessive mutation in a single gene. $^{2-4}$ AGE-1 is a subunit of the phosphoinositide 3-kinase (PI3K), and is a component of insulin/insulin-like growth factor-1 signaling (IIS) pathway, which is highly conserved in the animal kingdom. In some animals, IIS pathway has numerous insulin-like peptides (ILPs) as the ligands, compared with other signaling pathways. For example, C. elegans has 40 ILPs (Figure 1) as putative ligands for the sole insulin-like receptor, DAF-2..$^{5-7}$ Yet, why IIS pathway has numerous ligands to the sole receptor remains controversial. However, recent studies have revealed physiological functions of some ILPs. Here, we briefly review the structures, physiological functions, and secretion of $C$. elegans ILPs. We also show our recent studies with latest data.

\subsection{Identification and Structure of insulin-like peptides}

The small size of the $C$. elegans genome sequence (100 million base pairs; about the size of a single human chromosome) was completed in $1998 .^{8}$ The completion resulted in a tentative list of all the genes and a description of other sequence features. The information provided a powerful resource for reverse genetics such as RNA interference (RNAi) and gene disruption. ${ }^{9}$, 10 Together with forward/reverse genetics, developmental data, and anatomical data, it also provided major breakthroughs in the field of aging that will likely be made with $C$. elegans for two reasons. First, it has a very short lifespan (2-3 weeks at $\left.20^{\circ} \mathrm{C}\right)$, which can be tractable for genetic studies of aging. Second, some molecular mechanisms in C. elegans are conserved in other organisms. For example, mutations in daf-2 gene can increase the lifespan of active and fertile adults. The ability of daf-2 mutations to extend lifespan depends on the activity of daf-16 gene encoding a transcription factor, FOXO, autholog. ${ }^{11}$ This DAF-2 to DAF-16 pathway, called insulin/insulin-like growth factor-1 signaling (IIS) pathway, is highly conserved and involved in the regulation of lifespan among different organisms. ${ }^{12}$ Cloning of $d a f-2$ revealed that it encodes a receptor tyrosine kinase that shows more than $30 \%$ amino acid identity to the human insulin and IGF-I receptors. ${ }^{13}$ These discoveries attracted researchers working with C. elegans, including us, and awoke an interest in ligands for the DAF-2 receptor.

In 1998, we reported the identification of Ceinsulin-1 (ins-18) and Ceinsulin-2 (ins-17), 
Internal Biology Review. Volume 1, Issue 1, May 2017

\section{Diverse insulin-like peptides in Caenorhabditis elegans}

which encode insulin-like peptides (ILPs) in $C$. elegans, using Rapid amplication of cDNA end (RACE). ${ }^{14}$ Duret and co-workers have identified 3 new families of insulin-like peptides $(\alpha, \beta$, and $\gamma$ types) represented by 10 ILPs: $\gamma$ type ILP (INS-11), $\beta$ type ILPs (INS-2 through INS-7), and $\alpha$ type ILPs (INS-21 through INS-23). ${ }^{15} \gamma$ type ILPs have three canonical disulfide bonds (2 B-A bonds between $\mathrm{B}$ and $\mathrm{A}$ domains, 1 bond within $\mathrm{A}$ domain), the same as human insulin. $\beta$ type ILPs have an additional disulfide bond between $\mathrm{B}$ and $\mathrm{A}$ domains, in addition to the three disulfide bonds. $\alpha$ type ILPs have different patterns of three disulfide bonds (3 bonds between B and A domains). In 2001, Pierce and co-workers ${ }^{5}$ analyzed the entire $C$. elegans genomic sequence with multiple sequence searching tools, including BLAST, ${ }^{16}$ FASTA, ${ }^{17}$
Markoff model-based searches. $^{18}$ This procedure revealed 37 candidate insulin-like genes, ins-1 through ins-37. All of the 12 insulin-like genes (ins-2, 3, 4, 5, 6, 7, 11, 17, 18, 21,22 , and 23) identified previously ${ }^{14,15}$ were successfully detected in their research. $\mathrm{Li}$ and co-workers proposed that DAF-28, which is classified into the $\beta$ type ILP, functions as a ligand of DAF-2. ${ }^{6}$ Finally, an additional insulin-like peptide gene, ins-38, appeared to be present in the $C$. elegans genome (Steve Doberstein and Kim Ferguson, personal communication), while a novel insulin-like gene, ins-39, has been found by BLAST searches (Dhaval Patel and David Gems, personal communication). ${ }^{7}$ To data, the 40 ILPs, INS-1 INS-39, and DAF-28, have been identified, and are the putative ligands for DAF-2 receptor (Figure 1).
[ $\alpha$ type ILPs ]

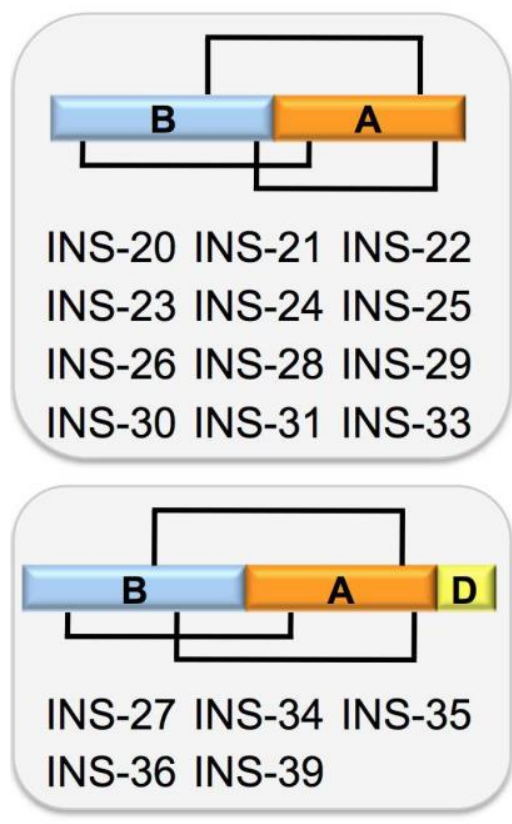

[ $\beta$ type ILPs ]

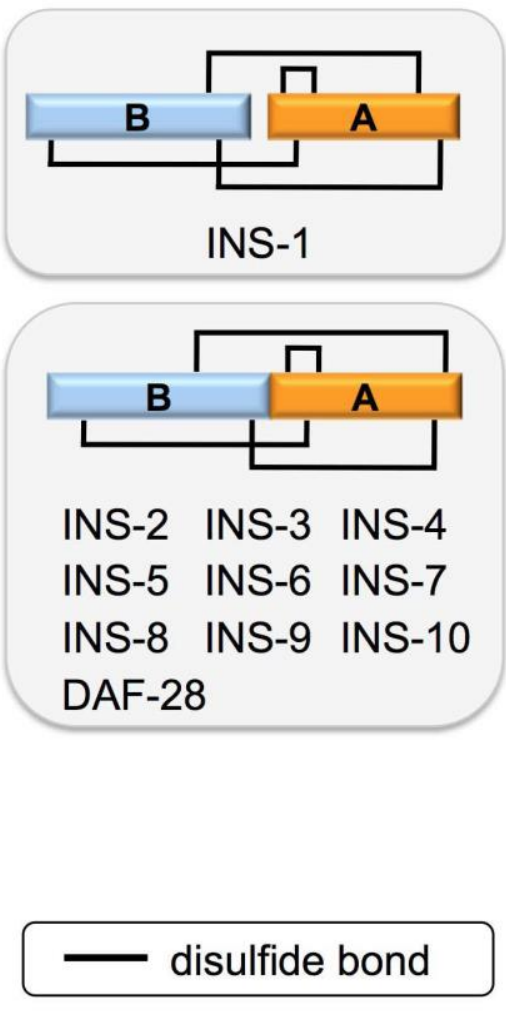

[ $\gamma$ type ILPs ]

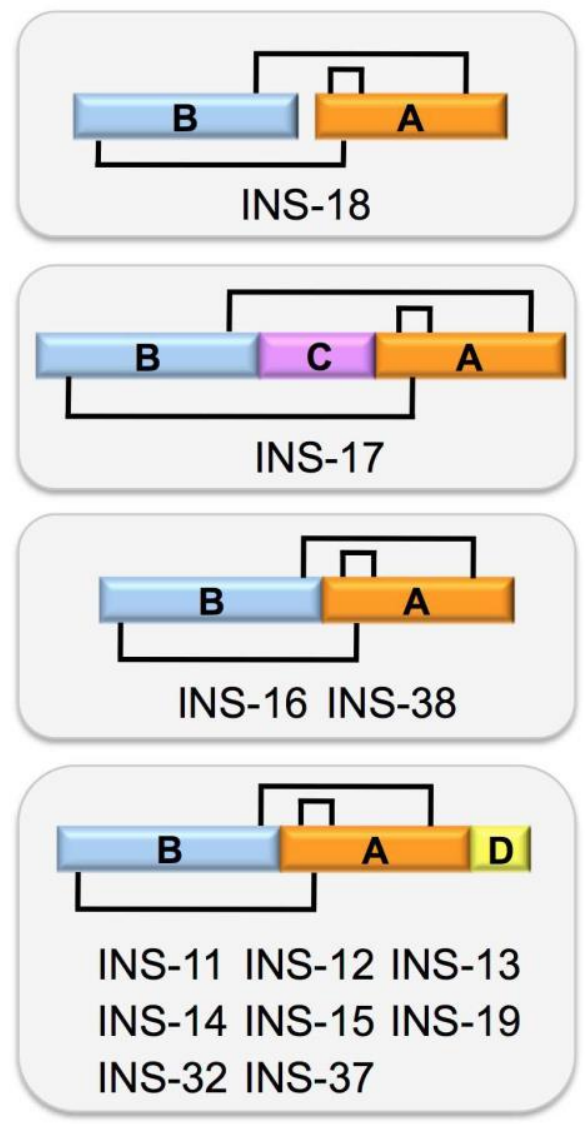

Figure 1: Schematic structures of 40 putative mature ILPs in C. elegans. 
Internal Biology Review. Volume 1, Issue 1, May 2017

\section{Diverse insulin-like peptides in Caenorhabditis elegans}

In mammals, insulin is first synthesized as a preproinsulin precursor, and then processed by multiple prohormone convertases (PCs) before release of the mature insulin, ${ }^{19,20}$ The process results in a B-A peptide linked by disulfide bonds. Some of $C$. elegans ILPs are also processed by PCs: three PC1 homologs, comprising AEX-5, BLI-4, and KPC-1, ${ }^{21,} 22$ and a PC2 homolog, EGK-3, ${ }^{23}$ to produce mature ILPs. ${ }^{24,25}$ Similar to mammalian insulin, the precursor of INS-18 is processed by KPC-1 (PC1 homolog) at the B-C junction and by EGL-3 (PC2 homolog) at the C-A junction, resulting in a $\mathrm{B}-\mathrm{A}$ peptide linked by disulfide bonds. In contrast, INS-1 is processed only by EGL-3 at the B-C and C-A junctions, since the C-peptide is flanked by two "KR" motives, which EGL-3 recognizes and proteolyzes. ${ }^{25}$ Other ILPs do not contain a C-peptide according to sequence analysis, indicating that INS-1 and INS-18 are structurally similar to insulin consisting of two polypeptides whereas other 38 ILPs are similar to IGF consisting of one polypeptide. Interestingly, among the $\beta$ type ILPs, INS-3, INS-4, and INS-6 are processed by EGL-3 whereas INS-2 and DAF-28 are processed by KPC-1 at the F-B junctions, resulting in a $\mathrm{B}-\mathrm{A}$ single strand peptide with disulfide bonds. ${ }^{24}$ C. elegans $\mathrm{PC} 1$ (KPC-1) is also necessary to promote the formation of higher order dendritic branches. ${ }^{26}$ Recently, it has been reported that mammalian PC1 (PCSK1) is downregulated in the induced pluripotent stem cell-derived (iPSC-derived) neurons from Prader-Willi syndrome (PWS) patients, indicating that neuroendocrine PWS-associated phenotypes are linked to reduced expression of PCSK1. ${ }^{27}$ Therefore, these studies of ILP processing are also relevant to neuronal diseases.

\subsection{Physiological functions}

Some researchers have reported physiological functions of ILPs in C. elegans. We demonstrated that RNAi knockdown or knockout of ins-18 results in a decrease in dauer formation and lifespan extension, whereas its overexpression results in an increase in dauer formation and lifespan extension. ${ }^{28,29}$ Activation of DAF-2, the sole $C$. elegans ILP receptor, initiates downstream phosphorylation of the kinase cascade, composed of AGE-1/PI3K, ${ }^{30}$ PDK-1/PDK, and ATKs (AKT-1 and -2), ${ }^{31,32}$ which leads to the phosphorylation and cytoplasmic retention of DAF-16, a FOXO type transcription factor. Partial loss of function of DAF-2 results in reduction of the phosphorylation and partial nuclear translocation of DAF-16, ${ }^{33-36}$ leading to an increase in dauer formation and lifespan extension. Therefore, INS-18 probably acts as an antagonistic ligand and suppresses DAF-2 activation. We also demonstrated that INS-12, INS-17, and INS-23, similar to INS-18, promote dauer formation and/or longevity. ${ }^{37-39}$ On the other hand, INS-7 and DAF-28, opposite to INS-18, act as agonistic ligands to suppress dauer formation and longevity. ${ }^{40-42}$

In $C$. elegans, IIS pathway is also essential to regulate postembryonic development, cell proliferation in germline, immunity, heat-resistance, and learning. ${ }^{43-48}$ For example, (1) mutation of ins- 1 showed defects in salt chemotaxis and thermotaxis learning. ${ }^{49,50}$ These researches provided new concepts for a study of memory-leaning associated with aging. Recently, it has been reported that one of $C$. elegans insulin-degrading enzymes (IDEs) is a key protein involved in Alzheimer's disease (AD). ${ }^{51}$ Thus, IIS in C. elegans is a good model to understand mechanisms in memory-leaning associated with aging and AD. (2)Loss of function of either ins-3 or ins-33 causes 
Internal Biology Review. Volume 1, Issue 1, May 2017

\section{Diverse insulin-like peptides in Caenorhabditis elegans}

reduction in the number of proliferative zone germ cells. In addition, loss of function of $d a f-2$ also cause the similar phenotype. ${ }^{46}$ In $C$. elegans, the GLP-1/Notch receptor pathway is known to maintain germ cells in the undifferentiated (mitotic) state and/or prevents their differentiation (meiotic entry). ${ }^{52}$ In mammalian intestinal stem cells, Notch signaling pathway also modulates proliferation and differentiation. ${ }^{53}$ Therefore, the $C$. elegans ILPs study could provide insights into stem cell research. (3)Fernandes de Abre and co-workers attempted to elucidate mechanisms for the forty C. elegans ILPs to coordinate diverse physiological processes, such as dauer formation, longevity, reproduction, thermotolerance, and pathogen resistance. ${ }^{54}$ This study revealed that 20 mutants in ins genes are susceptible to Pseudomonas aeruginosa. In contrast, ins-27 or ins-31 mutants show increased resistance to this pathogen. Moreover, ins-27 mutants also show an increase in thermotolerance $\left(34.5^{\circ} \mathrm{C}\right)$; smaller increases are found in ins-23 or ins-1 mutants. ${ }^{54}$

Taken together, IIS pathway is widely implicated in regulating diverse physiological processes from metabolism to longevity across many animal species. ILPs, as ligands, have significant roles in modulation of this pathway. Although diversity in functions of ILPs has been demonstrated, physiological functions of ILPs remains controversial. For example, Liu and co-workers found that INS-33 acts as antagonistic DAF-2 ligand, ${ }^{55}$ whereas Michaelson and co-workers implicated INS-33 as an agonistic ligand in the germ cell proliferation. $^{46}$
To elucidate ILPs functions (DAF-2 receptor agonist or antagonist against), we have developed a novel assay using $C$. elegans primary culture cells expressing DAF-16::GFP. ${ }^{56}$ As described above, DAF-16 is translocated from the cytoplasm to the nucleus in response to inactivation of DAF-2. Thus, we attempted to determine ILPs functions by intracellular localization of DAF-16::GFP. Briefly, to prepare an ILP, recombinant maltose binding protein (MBP)-INS-6 was expressed using pMAL-c $2 \mathrm{x}$ vector system and isolated by affinity chromatography with amylose resin. After refolding the MBP-INS-6 by gradual removal of urea and DTT, recombinant INS-6 was separated from MBP by factor Xa digestion. INS-6 has putative 4 disulfide bonds: Cys10-Cys39, Cys22-Cys52, Cys26-Cys53, and Cys38-Cys43 (Figure 2A). To investigate whether the purified INS-6 has the putative disulfide bonds pattern, we first performed MALDI-TOF-MS analysis. This analysis showed that 3 main peaks. One of them, a peak $(\mathrm{m} / \mathrm{z}=5655.06)$ is almost the same as calculated mass of INS-6 $(\mathrm{m} / \mathrm{z}=5655.45)$ (Figure 2B), meaning that recombinant INS-6 forms 4 pairs of intramolecular disulfide bonds without free cysteine residues.

Moreover, 3 major peaks $(\mathrm{m} / \mathrm{z}=$ $826.15,1146.92$, and 1225.44) were detected from the trypsin-digested recombinant INS-6 by MALDI-TOF-MS and ESI-MS analysis (Fig. $3 \mathrm{~A}$ and $\mathrm{B})$. These MS profiles correspond to calculated mass of putative fragments 1,2 , and 3 derived from trypsin-digested recombinant INS-6 (Figure 3C), suggesting that the recombinant INS- 6 has the putative 4 disulfide bonds. 
Internal Biology Review. Volume 1, Issue 1, May 2017

\section{Diverse insulin-like peptides in Caenorhabditis elegans}

\section{A}

Putative structure of INS-6
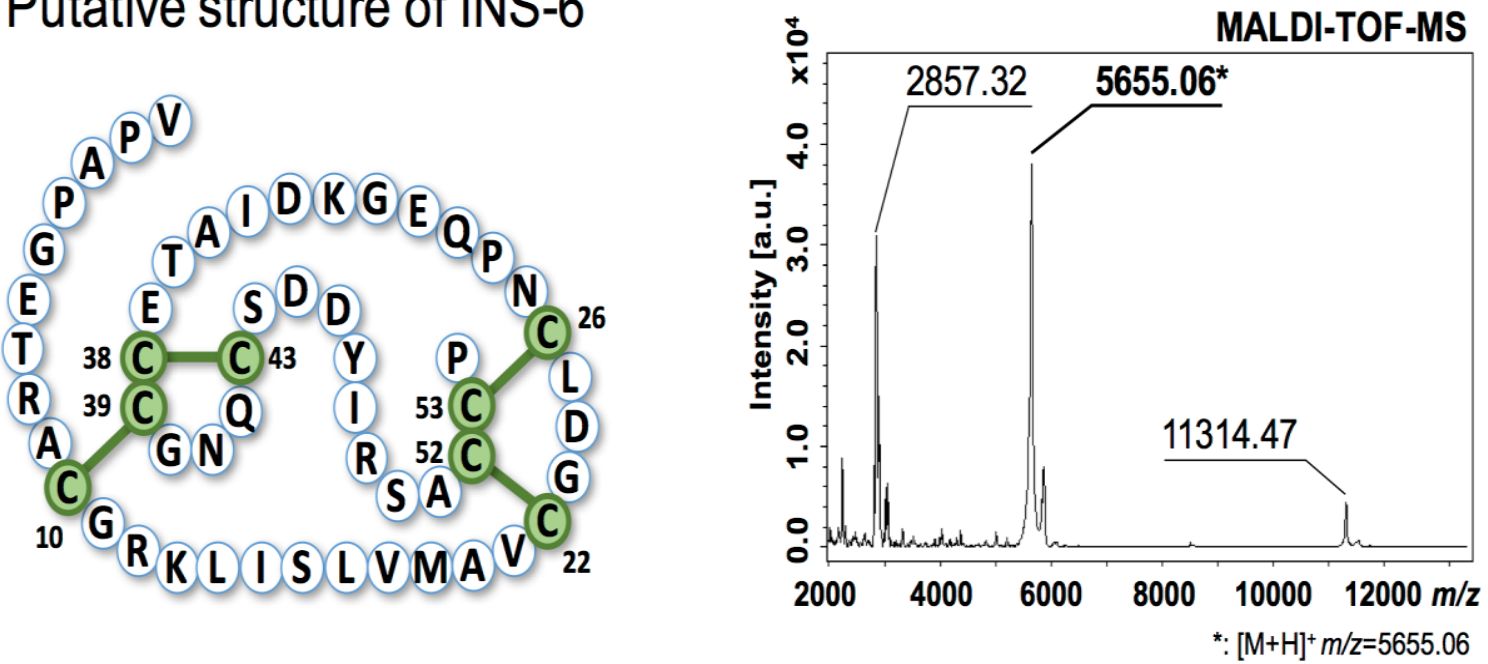

Figure 2: The recombinant INS-6 has 4 disulfide bonds. (A) Schematic structure of the recombinant INS-6. Greens indicate disulfide bonds. (B) MALDI-TOF-MS spectrum of the recombinant INS-6.

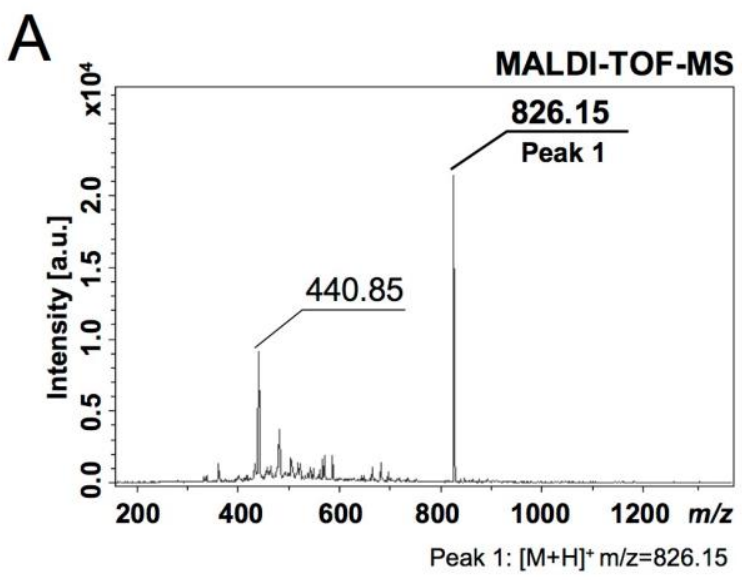

C

Putative fragmentation of INS-6

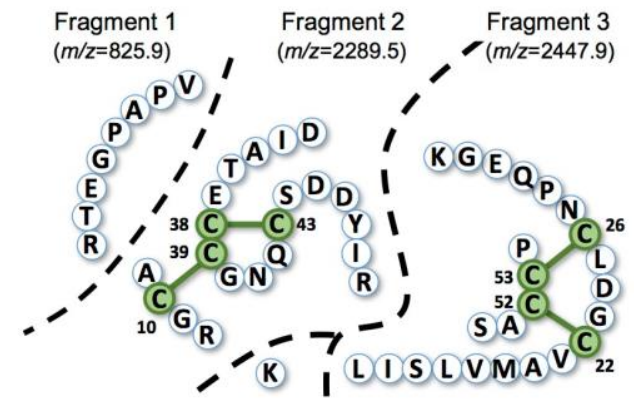

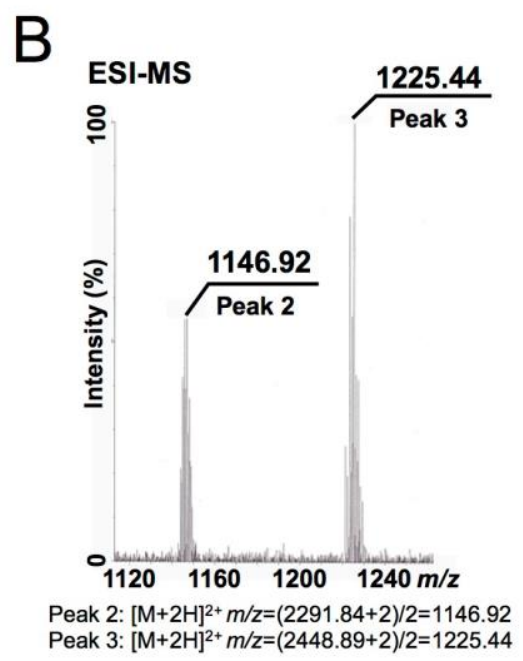

Figure 3: A putative pattern of 4 disulfide bonds in the recombinant INS-6. (A) MALDI-TOF-MS spectrum of the trypsin-digested recombinant INS-6. (B) ESI-MS spectrum of the trypsin-digested recombinant INS-6. (C) Schematic structure of putative fragmentation of the trypsin-digested recombinant INS-6. Greens indicate disulfide bonds. 


\section{Diverse insulin-like peptides in Caenorhabditis elegans}

Subsequently, the recombinant INS-6 was added to the primary culture cells derived from the worms expressing DAF-16::GFP to assess its function. Initially, DAF-16::GFP was localized in the nucleus probably due to its unphosphorylated form. After $10 \mathrm{~min}$. of the INS-6 treatment, translocation of DAF-16::GFP into cytoplasm was promoted (Figure 4). Moreover, this translocation is almost inhibited by daf-2 RNAi, suggesting that INS-6 acts as an agonist to activate DAF-2 receptor (Figure 4). Interestingly, although low relative to human or bovine insulin, INS-6 synthesized by solid-phase BOC-chemistry can binds to and activates the human insulin receptor. ${ }^{57}$ Therefore, our system provides insights into ILPs physiological functions.
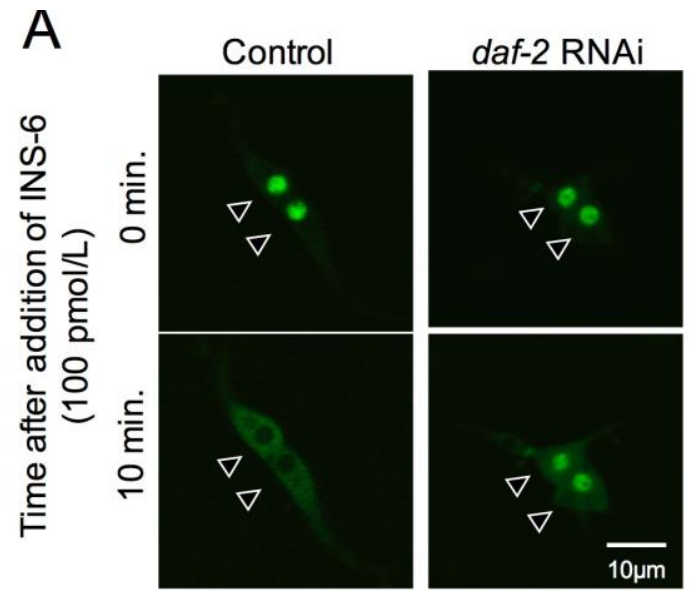

C

Primary culture cell with DAF-16::GFP

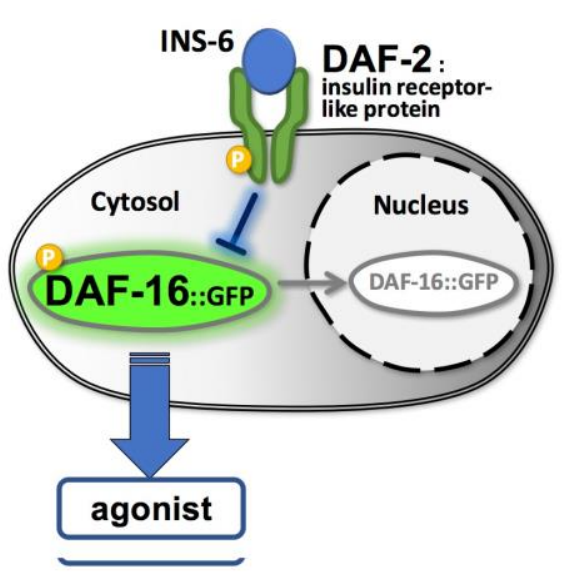

Figure 4: The recombinant INS-6 promotes the cytoplasmic translocation of DAF-16. (A) Localization of DAF-16::GFP in primary culture cells. Hollow arrowheads indicate cells with DAF-16::GFP. Scale bar is $10 \mu \mathrm{m}$. (B) Percentage of DAF-16::GFP positive cells treated with control RNAi ( $>$ 80); daf-2 RNAi ( $>40$ ). (C) Putative model suggests that the recombinant INS-6 functions as an agonist to promote the cytoplasmic translocation of DAF-16::GFP via DAF-2 receptor. 
Internal Biology Review. Volume 1, Issue 1, May 2017

\section{Diverse insulin-like peptides in Caenorhabditis elegans}

\subsection{Secretion}

Insulin is the major hormonal signal molecule for reducing blood glucose and storing energy in metabolites. In mammals, only the $\beta$-cells, located within 100-200 $\mu \mathrm{m}$ cell clusters (islets) throughout the pancreas, produce a significant amount of insulin. ${ }^{58}$ On the other hand, it has been reported that specialized thymic cells and the developing brain also produce insulin. ${ }^{59-61}$ C. elegans has "pseudocoelom", which is a fluid-filled body cavity lying inside its external body wall that bathes the internal organs, including the neuron, alimentary, and reproductive systems, respectively. The pseudocoelom functions as a lubricant between tissues, and provides a medium for intercellular signaling and nutrient transport, indicating this is positioned to carry out many of the functions normally performed by the circulatory or the respiratory systems in higher animals. Some ILPs are known to be secreted into the pseudocoelom and partly accumulate in coelomocytes. For example, DAF-28 is produced in the neuronal cells (ASI and ASJ) and secreted into pseudocoelom ${ }^{6,62}$; INS-35 is produced in the intestine and also secreted into pseudocoelom. $^{63}$ Areite-translocating ATPase (ASNA)-1 positively regulates these secretions. Interestingly, mammalian ASNA1 is expressed in pancreatic cells and promotes insulin release. ${ }^{62} \mathrm{GON}-1$, the $C$. elegans homolog of ADAMTS9, a metalloprotease that cleaves components of the extracellular matrix (ECM), ${ }^{64,65}$ also positively regulate INS-7 and DAF-28 (agonists) and INS-18 (an antagonist) secretions, suggesting that secretion of agonists and antagonists might not be differentially regulated. ${ }^{66}$ Because of fundamental similarities in the ILPs/insulin secretion mechanisms between the worms and humans, genetic and molecular pathways that control metabolism in C. elegans could be highly informative for analysis of human metabolic disease such as type-2 diabetes. Recently, we have found that the secretory polarity of INS-35 and INS-7 is changed in the intestinal epithelial cells, in response to growth conditions. These ILPs are normally secreted into the basal side. Their secretory polarity is changed into the apical side when worms undergo dauer arrest, larval diapause, under unfavorable growth conditions. On the contrary, these ILPs are secreted into the basal side again when the dauer larvae recover from diapause under favorable growth conditions (Figure 5). ${ }^{63}$ It is possible that molecular mechanism of sorting/packaging of the ILPs into secretory vesicles in normal development is different from that in dauer state. Formation of secretory vesicles is initiated in the trans-Golgi network (TGN). Secretory peptides are sorted and packaged into dense core vesicles there, resulting in formation of secretory granules. Then the granules are translocated onto cellular membranes. In this process of exocytosis, Rab family proteins, small $G$ proteins, play important roles. In insulin secretory granules, some proteins (channel, proton pump, ATPase, Rabs, etc) are identified by analyses using MS/MS. Among them, Rab3a, Rab27a have roles in the insulin exocytosis processes, such as a secretory vesicle regulator and a binding cortical actin rails. ${ }^{67}$ Therefore, a polarity of vesicle has already been determined after sorting/packaging. However, sorting/packaging mechanisms in TGN are not well understood. Thus, because of the availability of numerous genetic approaches, the ILPs research in $C$. elegans could provide useful information on regulatory mechanisms of sorting/packaging and secretion polarity. 


\section{Diverse insulin-like peptides in Caenorhabditis elegans}

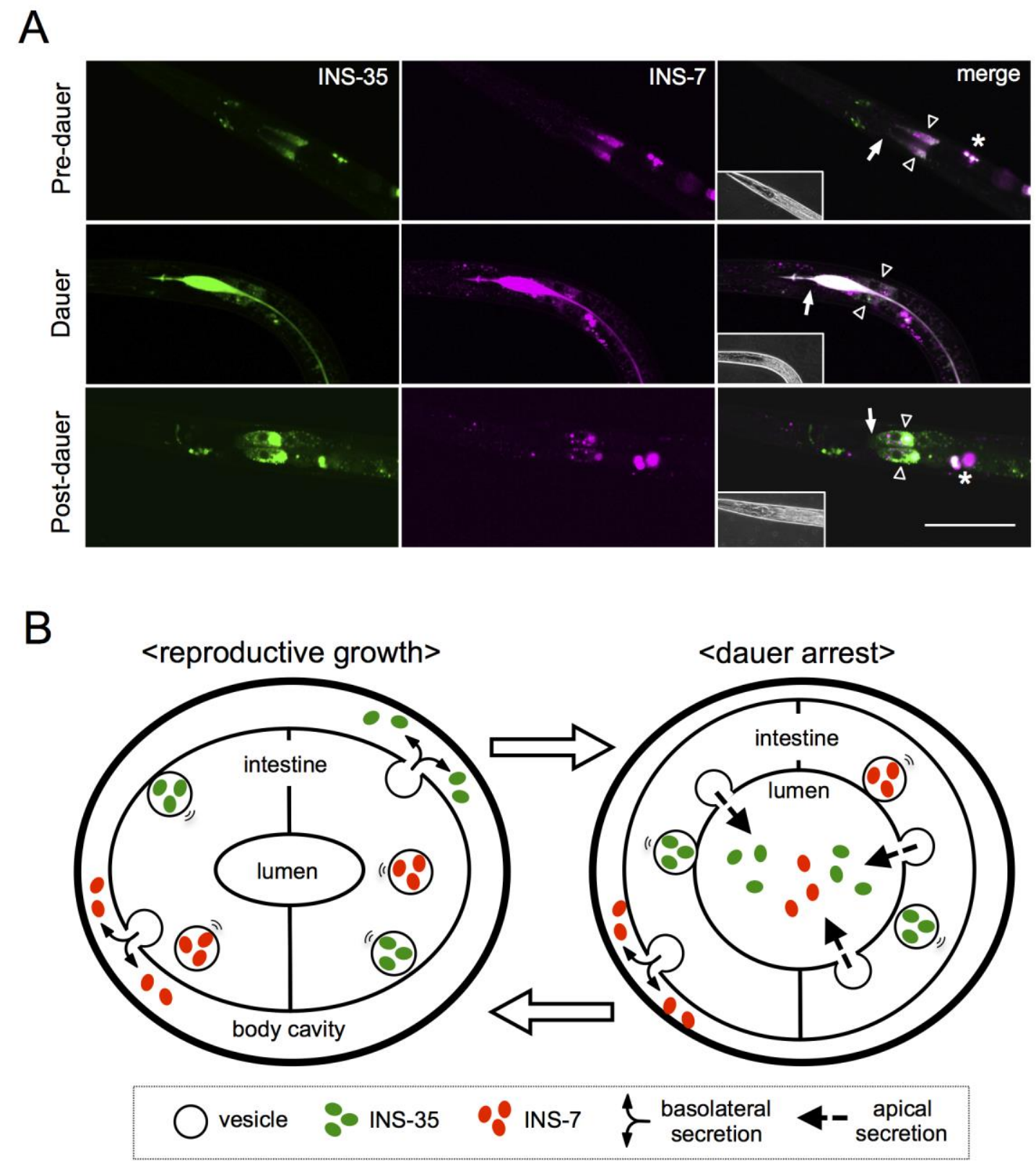

Figure 5: Polarity of secretion of INS-7 and INS-35 is changed between reproductive growth and dauer arrest. (A) Expression patterns of INS-35::VENUS (left), INS-7::mRFP (center) and merge (right) from pre-dauer to post-dauer stages. Hollow arrowheads indicate anterior intestinal cells. Arrows indicate the intestinal valves. Asterisks indicate coelomocytes. The intestinal canal is located between the intestinal cells (hollow arrowheads). The bright-field images are shown on the bottom left of the merged images. Scale bar, $50 \mathrm{~mm}$. (B) Model depicting a secretion switch between reproductive growth (left) and dauer arrest (right).

\section{Conclusion \& future direction}

We reviewed the processing to yield mature peptides, physiological functions, and secretion of $C$. elegans ILPs with our latest data. Despite the useful knowledge gleaned from the worm, there remain several unanswered 
Internal Biology Review. Volume 1, Issue 1, May 2017

\section{Diverse insulin-like peptides in Caenorhabditis elegans}

questions. Why are there agonistic and antagonistic ILPs? Do ILPs only function through the sole receptor tyrosine kinase? Several ILPs in C. elegans, INS-1 and INS-18, antagonize the DAF-2 signaling as either antagonists or partial agonists, resulting in the promotion of larval diapause and longevity. In the animal kingdom, endogenous antagonistic ILPs in other species have not yet reported so far. Why the worm has the antagonistic ILPs remains mysterious. It is most complicated that INS-1 acts as a DAF-2 antagonist in larval development whereas it acts as a DAF-2 agonist in salt chemotaxis and thermotaxis learning. ${ }^{5,}$ 4, 50 This contradiction is possibly caused by the splicing variation of $d a f-2 .{ }^{68}$ In fact, daf-2 has at least 6 splicing variants. Therefore, it is possible that the splicing variation of DAF-2 allow ILPs to modulate diverse biological processes. In addition, when and where ILPs are produced and secreted is also possibly relevant to their own physiological functions. The $C$. elegans genome sequencing revealed the sole insulin receptor tyrosine kinase, DAF-2. In mammals, the insulin binds to the insulin receptor (IR), which belongs to the large class of tyrosine kinase receptors whereas relaxin, one of the peptides in the insulin superfamily, binds to G-protein-coupled receptors (GPCRs). ${ }^{69}$ In starfish, a gonadotropic relaxin-like peptide, has been identified and its receptor has been suggested to be a GPCR. ${ }^{70,71}$ Therefore, it is possible that several $C$. elegans gonadotropic ILPs, such as INS-3 and INS-33, function through GPCRs.

Defects in the synthesis and secretion of peptide hormones cause several diseases such as diabetes. Therefore, it is an important subject to elucidate these molecular mechanisms. As we review, ILPs research in $C$. elegans has provided important insights, and could be a good platform for these researches.

\section{Acknowledgements:}

We are grateful to the C. elegans community for providing us with plasmids and mutant animals, and for fruitful discussions. We also thanks co-worker for their contribution to studies of C. elegans insulin-like peptides. Our studies described in this review were supported by the JSPS Kakenhi (Nos. 09265216, 11142207, 12760227, 14360208, 17580298, 20580112, 23580150, 26292061 to T.K.). 
Internal Biology Review. Volume 1, Issue 1, May 2017

\section{Diverse insulin-like peptides in Caenorhabditis elegans}

\section{References:}

1. Klass MR. A method for the isolation of longevity mutants in the nematode Caenorhabditis elegans and initial results. Mech Ageing Dev. 22 (3-4) 1993, 279-286.

2. Friedman DB, Johnson TE. A mutation in the age-1 gene in Caenorhabditis elegans lengthens life and reduces hermaphrodite fertility. Genetics. 118 (1) 1988, 75-86.

3. Friedman DB, Johnson TE. Three mutants that extend both mean and maximum life span of the nematode, Caenorhabditis elegans, define the age-1 gene. J. Gerontol. 43 (4) 1988, 102-109.

4. Johnson TE. Increased life-span of age-1 mutants in Caenorhabditis elegans and lower Gompertz rate of aging. Science. 249 (4971) 1990, 908-912.

5. Pierce SB, Costa M, Wisotzkey R, Devadhar $S$, Homburger SA, Buchman AR, Ferguson KC, Heller J, Platt DM, Pasquinelli AA, Liu LX, Doberstein SK, Ruvkun G. Regulation of DAF-2 receptor signaling by human insulin and ins-1, a member of the unusually large and diverse C. elegans insulin gene family. Genes Dev. 15 (6) 2001, 672-686.

6. Li W, Kennedy SG, Ruvkun, G. daf-28 encodes a $C$. elegans insulin superfamily member that is regulated by environmental cues and acts in the DAF-2 signaling pathway. Genes Dev. 17 (7) 2003, 844-858.

7. Husson SJ, Mertens I, Janssen T, Lindemans M, Schoofs L. Neuropeptidergic signaling in the nematode Caenorhabditis elegans. Prog Neurobiol. 82 (1) 2007, 33-55.

8. C. elegans Sequencing Consortium. Genome sequence of the nematode C. elegans: a platform for investigating biology. Science. 282 (5396) 1998, 2012-2018.

9. Fire A, Xu S, Montgomery MK, Kostas SA, Driver SE, Mello CC. Potent and specific genetic interference by double-stranded RNA in Caenorhabditis elegans. Nature. 391 (6669) 1998, 806-811.

10. Zwaal RR, Broeks A, van Meurs J, Groenen JT, Plasterk RH. Target-selected gene inactivation in Caenorhabditis elegans by using a frozen transposon insertion mutant bank. Proc Natl Acad Sci U S A. 90 (16) 1993, 7431-7435. 11. Kenyon C, Chang J, Gensch E, Rudner A, Tabtiang R. A C. elegans mutant that lives twice as long as wild type. Nature. 366 (6454) 1993, 461-464.

12. Barbieri $\mathrm{M}$, Bonafè $\mathrm{M}$, Franceschi $\mathrm{C}$, Paolisso G. Insulin/IGF-I-signaling pathway: an evolutionarily conserved mechanism of longevity from yeast to humans. Am J Physiol Endocrinol Metab. 285 (5) 2003, 1064-1071.

13. Kimura KD, Tissenbaum HA, Liu Y, Ruvkun G. daf-2, an insulin receptor-like gene that regulates longevity and diapause in Caenorhabditis elegans. Science. 277 (5328) 1997, 942-946.

14. Kawano T, Takuwa K, Nakajima T, Kimura Y. Insulin-like peptides of $C$. elegans. Worm Breeder's Gazette. 15 (2) 1998, 47.

15. Duret L, Guex N, Peitsch MC, Bairoch A. New insulin-like proteins with atypical disulfide bond pattern characterized in Caenorhabditis elegans by comparative sequence analysis and homology modeling. Genome Res. 8 (4) 1998, 348-353.

16. Altschul SF, Gish W, Miller W, Myers EW, Lipman DJ. Basic local alignment search tool. J Mol Biol. 215 (3) 1990, 403-410.

17. Pearson WR, Lipman DJ. Improved tools for biological sequence comparison. Proc Natl Acad Sci U S A. 85 (8) 1988, 2444-2448.

18. Eddy SR1, Mitchison G, Durbin R. Maximum discrimination hidden Markov models of sequence consensus. J Comput Biol. 2 (1) 1995, 9-23. 


\section{Diverse insulin-like peptides in Caenorhabditis elegans}

19. Orci L, Ravazzola M, Storch MJ, Anderson RG, Vassalli JD, Perrelet A. Proteolytic maturation of insulin is a post-Golgi event which occurs in acidifying clathrin-coated secretory vesicles. Cell. 49 (6) 1987, 865-868.

20. Malide D, Seidah NG, Chretien M, Bendayan M. Electron microscopic immunocytochemical evidence for the involvement of the convertases PC1 and PC2 in the processing of proinsulin in pancreatic beta-cells. J Histo Cyto. 43 (1) 1995, 11-19.

21. Thacker C, Rose AM. A look at the Caenorhabditis elegans Kex2/Subtilisin-like proprotein convertase family. Bioessays. 22 (6) 2000, 545-553.

22. Thacker C, Srayko M, Rose AM. Mutational analysis of bli- $4 / \mathrm{kpc}-4$ reveals critical residues required for proprotein convertase function in C. elegans. Gene. 252 (1-2) 2000, 15-25.

23. Kass J, Jacob TC, Kim P, Kaplan JM. The EGL-3 proprotein convertase regulates mechanosensory responses of Caenorhabditis elegans. J Neurosci. 21 (23) 2001, 9265-9272.

24. Hung WL, Hwang C, Gao S, Liao EH, Chitturi J, Wang Y, Li H, Stigloher C, Bessereau JL, Zhen M. Attenuation of insulin signalling contributes to FSN-1-mediated regulation of synapse development. EMBO J. 32 (12) 2013, 1745-1760.

25. Hung WL, Wang Y, Chitturi J, Zhen M. A Caenorhabditis elegans developmental decision requires insulin signaling-mediated neuron-intestine communication. Development. 141 (8) 2014, 1767-1779.

26. Salzberg Y, Ramirez-Suarez NJ, Bülow HE. The proprotein convertase KPC-1/furin controls branching and self-avoidance of sensory dendrites in Caenorhabditis elegans. PLoS Genet. 10 (9) 2014, e1004657.

27. Polex-Wolf J, Yeo GS, O'Rahilly S. Impaired prohormone processing: a grand unified theory for features of Prader-Willi syndrome? J Clin Invest. 127 (1) 2017, 98-99. 28. Kawano T, Ito $\mathrm{Y}$, Ishiguro $\mathrm{M}$, Takuwa $\mathrm{K}$, Nakajima T, Kimura Y. Molecular cloning and characterization of a new insulin/IGF-like peptide of the nematode Caenorhabditis elegans. Biochem Biophys Res Commun. 273 (2) 2000, 431-436.

29. Matsunaga Y, Gengyo-Ando K, Mitani S, Iwasaki T, Kawano T. Physiological function, expression pattern, and transcriptional regulation of a Caenorhabditis elegans insulin-like peptide, INS-18. Biochem Biophys Res Commun. 423 (3) 2012, 478-483.

30. Morris JZ, Tissenbaum HA, Ruvkun G. A phosphatidylinositol-3-OH kinase family member regulating longevity and diapause in Caenorhabditis elegans. Nature. 382 (6591) 1996, 536-539.

31. Paradis S, Ruvkun G. Caenorhabditis elegans Akt/PKB transduces insulin receptor-like signals from AGE-1 PI3 kinase to the DAF-16 transcription factor. Genes Dev. 12 (16) 1998, 2488-2498.

32. Paradis S, Ailion M, Toker A, Thomas JH, Ruvkun G. A PDK1 homolog is necessary and sufficient to transduce AGE-1 PI3 kinase signals that regulate diapause in Caenorhabditis elegans. Genes Dev. 13 (11) 1999, 1438-1452. 33. Ogg S, Paradis S, Gottlieb S, Patterson GI, Lee L, Tissenbaum HA, Ruvkun G. The Fork head transcription factor DAF-16 transduces insulin-like metabolic and longevity signals in C. elegans. Nature. 389 (6654) 1997, 994-999.

34. Henderson ST, Johnson TE. daf-16 integrates developmental and environmental inputs to mediate aging in the nematode Caenorhabditis elegans. Curr Biol. 11 (24) 2001, 1975-1980.

35. Lee RY, Hench J, Ruvkun G. Regulation of C. elegans DAF-16 and its human ortholog 


\section{Diverse insulin-like peptides in Caenorhabditis elegans}

FKHRL1 by the daf-2 insulin-like signaling pathway. Curr Biol. 11 (24) 2001, 1950-1957. 36. Lin $\mathrm{K}$, Hsin H, Libina N, Kenyon C. Regulation of the Caenorhabditis elegans longevity protein DAF-16 by insulin/IGF-1 and germline signaling. Nat Genet. 28 (2) 2001, 139-145.

37. Matsunaga $\mathrm{Y}$, Ito $\mathrm{H}$, Kawano $\mathrm{T}$. Proceedings: Physiological Function of INS-12, One of the Type- $\gamma$ Insulin-like peptides, in $C$. elegans. Peptide Science 2009. 2010, 459-462.

38. Matsunaga Y, Nakajima K, Gengyo-Ando K, Mitani S, Iwasaki T, Kawano T. A Caenorhabditis elegans insulin-like peptide, INS-17: its physiological function and expression pattern. Biosci Biotechnol Biochem. 76 (11) 2012, 2168-2172.

39. Matsukawa T, Matsunaga $Y$, Iwasaki $T$, Nagata K, Tanokura M, Kawano T. Proceedings: Comparison of physiological function between antagonistic insulin-like peptides, INS-23 and INS-18, in Caenorhabditis elegans. Peptide Science 2016. 2017, in press.

40. Malone EA, Inoue T, Thomas JH. Genetic analysis of the roles of daf-28 and age-1 in regulating Caenorhabditis elegans dauer formation. Genetics. 143 (3) 1996, 1193-1205. 41. Murphy CT, McCarroll SA, Bargmann CI, Fraser A, Kamath RS, Ahringer J, Li H, Kenyon C. Genes that act downstream of DAF-16 to influence the lifespan of Caenorhabditis elegans. Nature. 424 (6946) 2003, 277-283.

42. Cornils A, Gloeck M, Chen Z, Zhang Y, Alcedo J. Specific insulin-like peptides encode sensory information to regulate distinct developmental processes. Development. 138 (6) 2011, 1183-1193.

43. Kurz CL, Tan MW. Regulation of aging and innate immunity in C. elegans. Aging Cell. 3 (4) 2004, 185-193.
44. Kaletsky R, Murphy CT. The role of insulin/IGF-like signaling in $C$. elegans longevity and aging. Dis Model Mech. 3 (7-8) 2010, 415-419.

45. Kenyon C. A pathway that links reproductive status to lifespan in Caenorhabditis elegans. Ann N Y Acad Sci. 2010 Aug;1204 2010, 156-162.

46. Michaelson D, Korta DZ, Capua Y, Hubbard EJ. Insulin signaling promotes germline proliferation in C. elegans. Development. 137 (4) 2010, 671-680

47. Tissenbaum HA. Genetics, life span, health span, and the aging process in Caenorhabditis elegans. J Gerontol A Biol Sci Med Sci. 67 (5) 2012, 503-510.

48. Sasakura H, Mori I. Behavioral plasticity, learning, and memory in C. elegans. Curr Opin Neurobiol. 23 (1) 2013, 92-99.

49. Kodama E, Kuhara A, Mohri-Shiomi A, Kimura KD, Okumura M, Tomioka M, Iino Y, Mori I. Insulin-like signaling and the neural circuit for integrative behavior in C. elegans. Genes Dev. 20 (21) 2006, 2955-2960.

50. Tomioka M, Adachi T, Suzuki H, Kunitomo H, Schafer WR, lino Y. The insulin/PI 3-kinase pathway regulates salt chemotaxis learning in Caenorhabditis elegans. Neuron. 51 (5) 2006, 613-625.

51. Haque R, Nazir A. Identification and functional characterization of a putative IDE, C28F5.4 (ceIDE-1), in Caenorhabditis elegans: Implications for Alzheimer's disease. Biochim Biophys Acta. 1860 (11 Pt A) 2016, 2454-2462.

52. Austin J, Kimble J. $g l p-1$ is required in the germ line for regulation of the decision between mitosis and meiosis in C. elegans. Cell. 51 (4) 1987, 589-599.

53. VanDussen KL, Carulli AJ, Keeley TM, Patel SR, Puthoff BJ, Magness ST, Tran IT, Maillard I, Siebel C, Kolterud Å, Grosse AS, 


\section{Diverse insulin-like peptides in Caenorhabditis elegans}

Gumucio DL, Ernst SA, Tsai YH, Dempsey PJ, Samuelson LC. Notch signaling modulates proliferation and differentiation of intestinal crypt base columnar stem cells. Development. 139 (3) 2012, 488-497.

54. Fernandes de Abreu DA, Caballero A, Fardel P, Stroustrup N, Chen Z, Lee K, Keyes WD, Nash ZM, López-Moyado IF, Vaggi F, Cornils A, Regenass M, Neagu A, Ostojic I, Liu C, Cho Y, Sifoglu D, Shen Y, Fontana W, Lu H, Csikasz-Nagy A, Murphy CT, Antebi A, Blanc E, Apfeld J, Zhang Y, Alcedo J, Ch'ng Q. An insulin-to-insulin regulatory network orchestrates phenotypic specificity in development and physiology. PLoS Genet. 201410 (3) 2014, e1004225.

55. Liu T, Zimmerman KK, Patterson GI. Regulation of signaling genes by TGFbeta during entry into dauer diapause in $C$. elegans. BMC Dev Biol. 4 (11) 2004, 1-17.

56. Iwasaki $T$, Komatsu $M$, Fujimori $T$, Kawano T. Proceedings: Functional analysis of INS-6, one of the insulin-like peptides in $C$. elegans using primary culture. Peptide Science 2011. 2012, 369-372.

57. Hua QX, Nakagawa SH, Wilken J, Ramos RR, Jia W, Bass J, Weiss MA. A divergent INS protein in Caenorhabditis elegans structurally resembles human insulin and activates the human insulin receptor. Genes Dev. 17 (7) 2003, 826-831.

58. Suckale J, Solimena M. Pancreas islets in metabolic signaling focus on the beta-cell. Front Biosci. May (13) 2008, 7156-7171.

59. Derbinski J, Schulte A, Kyewski B, Klein L. Promiscuous gene expression in medullary thymic epithelial cells mirrors the peripheral self. Nat Immunol. 2 (11) 2001, 1032-1039.

60. Deltour L, Leduque P, Blume N, Madsen O, Dubois P, Jami J, Bucchini D. Differential expression of the two nonallelic proinsulin genes in the developing mouse embryo. Proc Natl Acad Sci U S A. 90 (2) 2003, 527-531.

61. Devaskar SU, Giddings SJ, Rajakumar PA, Carnaghi LR, Menon RK, Zahm DS. Insulin gene expression and insulin synthesis in mammalian neuronal cells. J Biol Chem. 269 (11) 1994, 8445-8454.

62. Kao G, Nordenson C, Still M, Rönnlund A, Tuck S, Naredi P. ASNA-1 positively regulates insulin secretion in C. elegans and mammalian cells. Cell. 128 (3) 2007, 577-587.

63. Matsunaga Y, Honda Y, Honda S, Iwasaki T, Qadota H, Benian GM, Kawano T. Diapause is associated with a change in the polarity of secretion of insulin-like peptides. Nat Commun. Feb (7) 2016, 10573.

64. Clark ME, Kelner GS, Turbeville LA, Boyer A, Arden KC, Maki RA. ADAMTS9, a novel member of the ADAM-TS/ metallospondin gene family. Genomics. 67(3) 2000, 343-350.

65. Somerville RP, Longpre JM, Jungers KA, Engle JM, Ross M, Evanko S, Wight TN, Leduc R, Apte SS. Characterization of ADAMTS-9 and ADAMTS-20 as a distinct ADAMTS subfamily related to Caenorhabditis elegans GON-1. J Biol Chem. 2278 (11) 2003, 9503-9513.

66. Yoshina S, Mitani S. Loss of C. elegans GON-1, an ADAMTS9 Homolog, Decreases Secretion Resulting in Altered Lifespan and Dauer Formation. PLoS One. 10 (7) 2015, e0133966

67. Suckale J, Solimena M. The insulin secretory granule as a signaling hub. Trends Endocrinol Metab. 21 (10) 2010, 599-609.

68. Tomioka M, Naito Y, Kuroyanagi H, Iino Y. Splicing factors control C. elegans behavioural learning in a single neuron by producing DAF-2c recdeptor. Nat Commun. 7 2016, 11645. 
Internal Biology Review. Volume 1, Issue 1, May 2017

\section{Diverse insulin-like peptides in Caenorhabditis elegans}

69. Hsu SY, Nakabayashi K, Nishi S, Kumagai J, Kudo M, Sherwood OD, Hsueh AJ. Activation of orphan receptors by the hormone relaxin. Science. 295 (5555) 2002, 671-674.

70. Mita M, Yoshikuni M, Ohno K, Shibata Y, Paul-Prasanth B, Pitchayawasin S, Isobe M, Nagahama Y.A relaxin-like peptide purified from radial nerves induces oocyte maturation and ovulation in the starfish, Asterina pectinifera. Proc Natl Acad Sci U S A. 106 (23) 2009, 9507-9512.

71. Mita M, Haraguchi S, Watanabe M, Takeshige Y, Yamamoto K, Tsutsui K. Involvement of Gas-proteins in the action of relaxin-like gonad-stimulating substance on starfish ovarian follicle cells. Gen Comp Endocrinol. 205 2014, 80-87. 